Vari C.E. ${ }^{1}$, Suciu H. ${ }^{2}$, Ispas Mihaela², Voidăzan S. ${ }^{3}$, Muntean Daniela Lucia ${ }^{4}$

\title{
Immunosuppressant medication in cardiac transplant patients - between therapeutic benefit and iatrogenic pathology
}

\author{
${ }^{1}$ Pharmacology and Clinical Pharmacy Department \\ ${ }^{2}$ Institute of Cardiovascular Diseases and Transplantation Târgu Mureş \\ ${ }^{3}$ Epidemiology Department \\ ${ }^{4}$ Drug analysis Department \\ University of Medicine and Pharmacy Târgu Mureș
}

\begin{abstract}
ABTRACT.
In a retrospective study, the safety profile and efficacy of immunosuppressive medication was evaluated in 35 patients with cardiac transplant in Targu Mures during 1999-2011 (11 treated with cyclosporine, 24 with tacrolimus, both drugs were associated with mycophenolate mofetil). Therapeutic benefit was measured by survival curve and lack of rejection while safety was evaluated by measuring plasma immunosuppressive drugs levels and evaluation of specific adverse events (nephrotoxicity, diabetes, and hypertension). The most frequent side effect was nephrotoxicity (significant reduction in glomerular filtration rate estimated by MDRD formula), but no significant differences were found between the 2 medications.
\end{abstract}

Keywords: cyclosporine, tacrolimus, side effects, heart transplant

Suciu H

University of Pharmacy and Medicine Tg. Mures

$38 \mathrm{Gh}$. Marinescu, Tg. Mureş,

Tel: +40-265-215-551

E-mail: horisuciu@gmail.com

\section{Introduction}

Introduction of specific immunosuppressive drug therapy (calcineurin inhibitors, rapamycine analogues) allowed the possibility of performing large scale organ transplant; despite the increase in survival and a relatively good safety, iatrogenic risk and drug interactions of clinical significance remain important. If the risk of high blood pressure and diabetes can be drug controlled, nephrotoxicity and risk of rejection complications remain of high importance.

\section{Premises and purpose of study}

Calcineurin inhibitors (cyclosporine, tacrolimus) present several proprieties that limit their usefulness and make long-term therapy not a viable option in the absence of monitoring blood levels by pharmacokinetic criteria (required by clinical guidelines) $[1,2,6]$, as follows:

- narrow therapeutic index (which requires maintaining plasma level in the limits imposed by clinical guidelines specific to each type of transplant) to avoid underdosage (favoring rejection) or overdosage 
(risk of bacterial, viral or fungal infection);

- intrinsic high chronic toxicity, especially nephrotoxicity iatrogenic induced already obvious after the first years post-transplant;

- The risk of hypertension and impaired glucose metabolism leading to iatrogenic diabetes, which are associated comorbidities requiring concomitant treatment and promote further deterioration of renal function.

Nephrotoxicity of cyclosporine and tacrolimus is well known, being described both in terms of acute toxicity (vasoconstriction and decrease of glomerulare filtration rate due to activation of the renin-angiotensin -aldosterone system, increase of endothelin levels and decrease in the formation of prostaglandins, prostacyclin and NO) as well as chronic toxicity often the cause of late rejection and the occurrence of chronic kidney disease in patients with non-renal transplantation $[7,8]$.

Chronic toxicity is dose-dependent, while the occurrence of chronic renal disease is related to the length of drug administration, which corresponds to the total cumulative dose. Moreover, chronic renal disease modifies the pharmacokinetics of drugs and the decreased renal clearance causes a vicious circle by increasing blood levels and drug half-life, which impose new dose adjustment and close monitoring (therefore, even in the absence of drug interactions and establishment of a correct individual pharmacokinetic profile, dose should be adjusted periodically).

\section{Materials and Methods}

The study was retrospective, evaluating the therapeutic benefit (in terms of survival post transplant) and specific adverse reactions of immunosuppressive medication.

The following parameters were monitored:

- Blood levels of cyclosporine and tacrolimus and their classification in the recommended range by clinical guidelines on heart transplant; as a pharmacokinetic marker residual concentration $(\mathrm{C} 0$ - through concentration) was used.

- Renal function, estimated in time (posttransplant) as a marker of immunosuppressive drug nephrotoxicity by quantifying glomerular filtration rate (using MDRD equation - Modified Diet on Renal Disease to calculate endogenous creatinine clearance) $[3,4]$;

- Development or worsening of co-morbidities (diabetes, viral, bacterial or fungal infections) likely to be classified as iatrogenic adverse effects caused by immunosuppressive status;

- Evaluating clinical consequences of posttransplant immunosuppressive therapy (chronic rejection, dose-dependent toxicity, (i)reversible cumulative toxic effects - hypertension, diabetes mellitus, chronic renal disease).

Plasma levels of cyclosporine and tacrolimus from 35 patients with heart transplant performed at the transplant center of Targu Mures in the period 1999-2011 were used (age between 16-59 years, the average being $37.5 \pm 14,1$ years, 11 treated with cyclosporine, 24 treated with tacrolimus, both drugs were associated with mycophenolate mofetil).

For statistical analysis of the results, ANOVA test was used and differences were considered significant when $\mathrm{p}$ value was less than 0.05 .

\section{Results. Analysis of therapeutic benefit}

Therapeutic range - Recommended blood concentrations for the 2 immunosuppressive drugs in cardiac transplantation [5] are presented in Table no. I. 
Table no. I - Targeted values for plasma concentration of calcineurin inhibitors in heart transplantation

\begin{tabular}{|c|c|c|c|}
\hline \multirow{2}{*}{$\begin{array}{c}\text { Immunosuppressant } \\
\text { drug }\end{array}$} & \multicolumn{2}{|c|}{$\begin{array}{c}\text { Target values } \\
\text { (whole blood) }\end{array}$} & \multirow{2}{*}{ Comments } \\
\cline { 2 - 4 } & $\begin{array}{c}\mathrm{C}_{0} \\
(\mathrm{ng} / \mathrm{mL})\end{array}$ & $\begin{array}{c}\mathrm{C}_{2} \\
(\mathrm{mg} / \mathrm{L})\end{array}$ & \\
\hline \multirow{2}{*}{ Tacrolimus } & $10-20$ & - & $\begin{array}{c}\text { The first } 3 \\
\text { months }\end{array}$ \\
\cline { 2 - 4 } & $5-15$ & - & $\begin{array}{c}4 \text { months } \\
-1 \text { year post- } \\
\text { transplant }\end{array}$ \\
\hline \multirow{2}{*}{ Cyclosporine } & $250-350$ & - & \begin{tabular}{c} 
initially \\
\cline { 2 - 4 }
\end{tabular} \\
\cline { 2 - 4 } & $100-200$ & $0.3-0.6$ & $\begin{array}{c}6 \text { months } \\
1 \text { year post- } \\
\text { transplant }\end{array}$ \\
\hline
\end{tabular}

Values obtained in the period up to 5 years post-transplant for the group considered are presented in Table no.2.

Average survival in patients receiving tacrolimus was $6.33 \pm 0.43$ years $(95 \%$ confidence interval - [5.47 - 7.19], with 2 deaths during followup) and average survival in patients receiving cyclosporine was $8.4 \pm 1.14$ years $(95 \%$ confidence interval - [6.20 -10.68], with 5 deaths during followup) survival rate at 3 years was $91.7 \%$ (see Fig. 1)

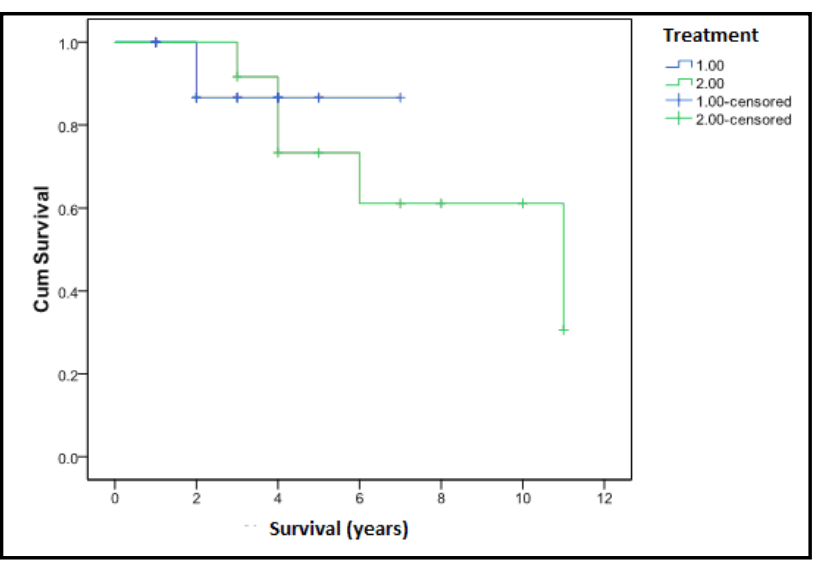

Figure 1 - Kaplan Meyer survival curve according to immunosuppressive therapy administered (1-tacrolimus, cyclosporine 2)

\section{The analysis of drug iatrogeny will be presented on levels of interest, as follows:}

Hypertension and risk of infection. These side effects are unavoidable on the long term and were present practically in all patients studied. These reactions are predictable; they can be managed by choosing targeted corrective medication. In addition to individualized clinical criteria, the compatibility with the degree of renal impairment and avoidance of pharmacokinetic drug interactions (metabolism via CYP450 isoform CYP3A4 / 5) were also taken into account.

Nephrotoxicity. During 5 years, compared to baseline glomerular filtration rate decreased significantly, in both treatments (cyclosporine, tacrolimus respectively); evolution towards chronic renal disease based on staging criteria imposed by the American Kidney Association (K / DOQI - Kidney Disease Outcome Quality initiative) is obvious. Nephrotoxicity imposed gradual adjustment of required dosage of calcineurin inhibitors - glomerular filtration rate was significantly reduced at 3 months post-transplant, the degradation was obvious after 5 years $(29.9 \pm 4.06$ vs. $126.8 \pm 50.16 \mathrm{ml} / \mathrm{min} / 1.73$ $\mathrm{m} 2$ for tacrolimus group, respectively $65.5 \pm 36.8 \mathrm{vs}$. $99.7 \pm 15.83 \mathrm{ml} / \mathrm{min} / 1.73 \mathrm{~m} 2$ for cyclosporine group). Between the 2 treatments there are no significant statistic differences both at baseline - at one month post-transplant $(\mathrm{p}=0.16)$ and after 5 years of follow up $(\mathrm{p}=0.25)$, due to large dispersion of results .

Iatrogenic diabetes. Cyclosporine and tacrolimus produce hyperglycemia, in the studied group diabetes was determined in 9 patients from 35 at 6 months after transplantation (de novo diabetes). 
Table no. 2 - Plasma values for calcineurin inhibitors in the studied group with cardiac transplantation

\begin{tabular}{|c|c|c|c|c|c|c|c|c|}
\hline \multirow{2}{*}{$\begin{array}{l}\text { Immunosuppressant } \\
\text { drug }\end{array}$} & \multicolumn{8}{|c|}{$\begin{array}{l}\text { Through concentration } \mathrm{C}_{0} \pm \mathrm{DS}(\mathrm{ng} / \mathrm{mL}) \\
\text { (whole blood) }\end{array}$} \\
\hline & 1 month & 3 months & 6 months & 1 year & 2 years & 3 years & 4 years & 5 years \\
\hline \multirow{2}{*}{ Tacrolimus } & $\begin{array}{c}11.85 \pm \\
\mathbf{3 . 5 4}\end{array}$ & $\begin{array}{c}10.34 \pm \\
3.86\end{array}$ & $\begin{array}{c}11.21 \pm \\
2.33\end{array}$ & $\begin{array}{l}11.11 \pm \\
\mathbf{2 . 8 0}\end{array}$ & $\begin{array}{c}9.16 \pm \\
2.17\end{array}$ & $\begin{array}{c}13.88 \pm \\
\mathbf{5 . 6 2}\end{array}$ & $\begin{array}{c}11.84 \pm \\
\mathbf{4 . 1 8}\end{array}$ & - \\
\hline & $\mathrm{n}=21$ & $\mathrm{n}=17$ & $\mathrm{n}=19$ & $\mathrm{n}=16$ & $\mathrm{n}=11$ & $\mathrm{n}=6$ & $\mathrm{n}=5$ & - \\
\hline \multirow{2}{*}{ Cyclosporine } & $\begin{array}{r}301.7 \pm \\
\mathbf{1 0 3 . 9 9} \\
\end{array}$ & $\begin{array}{r}223.8 \pm \\
\mathbf{1 0 1 . 4 8}\end{array}$ & $\begin{array}{c}242.2 \pm \\
\mathbf{5 5 . 6 7}\end{array}$ & $\begin{array}{r}201.6 \pm \\
\mathbf{1 0 6 . 3 4} \\
\end{array}$ & $\begin{array}{c}182.9 \pm \\
\mathbf{4 6 . 4 7}\end{array}$ & $\begin{array}{c}155.2 \pm \\
\mathbf{7 0 . 6 0}\end{array}$ & $\begin{array}{c}121.8 \pm \\
\mathbf{3 7 . 3 2}\end{array}$ & $\begin{array}{c}114.6 \pm \\
\mathbf{4 8 . 4 9}\end{array}$ \\
\hline & $\mathrm{n}=12$ & $\mathrm{n}=12$ & $\mathrm{n}=11$ & $\mathrm{n}=13$ & $\mathrm{n}=11$ & $\mathrm{n}=9$ & $\mathrm{n}=5$ & $\mathrm{n}=5$ \\
\hline
\end{tabular}

\section{Discussion}

Although described for a long time, nephrotoxicity of calcineurin inhibitors is still the subject of controversy. Small size of the study group (35 patients after heart transplantation, 11 treated with cyclosporine, 24 with tacrolimus) did not evidence significant differences between renal pharmacotoxicological potential of the 2 immunosuppressive drugs, which was described by Ispas $\mathrm{M}$ et al on our studied group [9]. Moreover, glomerular filtration rate at one month post transplantation, in case of immunosuppressive treatment is negatively correlated $(r=-0.63)$ with high statistical significance $(\mathrm{p}<0.0001)$ with patient age, which is an important predictor of renal function deterioration [9].

Superiority of tacrolimus compared to cyclosporine in terms of pharmacotoxicology is still controversial. Castel MA et al. (2011) shows that tacrolimus significantly increases the survival rate compared to cyclosporine in cardiactransplant patients, but the rejection rate was similar [10]. Moreover, the combination of tacrolimus - mycophenolate mofetil was superior to the combination of cyclosporine mofetil for prevention of acute rejection, by achieving target concentrations of mycophenolic acid (the active metabolite of mycophenolate mofetil) with lower doses of the parent drug, according to the results obtained by Meiser BM et al. (2004) [11]. However, the trial TICTAC ("tacrolimus in combination, tacrolimus alone compared trial") led by Baran DA et al (2011) shows that this combination (tacrolimus + mycophenolate) is not superior to monotherapy [12], but monotherapy is not possible in all patients and can be dangerous if plasma levels are suboptimal in non-compliant patients, in Kobashigawa JA's opinion [13].

Martins L. et al. (2004) showed that the transfer of renal transplant patients ininially treated with cyclosporine to tacrolimus after at least 6 months of treatment is correlated with improved renal function, suggesting a lower tacrolimus nephrotoxicity. The same authors have shown that the incidence of iatrogenic diabetes is similar in the two cases (about 20\%). [7]. Tacrolimus seems to have a similar or greater diabetogenic risk than cyclosporine (Garlicki M, 2005), but a lower cardiovascular risk (hypertension, lipid profile) [14]. Transfer from ciclosporin to tacrolimus appears to improve kidney function and lower risk of hypertension (in terms of giving up corticosteroids shortly after transplantation and association of statins) in pediatric cardiac transplant (Simmonds J, 2009) [15]

In case of kidney transplants, Morales JM et al (2005) presented that on long-term (10 years), the safety and the efficacy of tacrolimus compared with cyclosporine is superior, measurable in a very good functioning of the graft, no proteinuria and a normal value plasma creatinine [16]. 
Our results confirm that patient age at the time of transplantation, the presence or development of diabetes are predictors associated with progressive deterioration of renal function, which was already highlighted by Ojo AO et al. (2003) [8].

\section{Eonclusions}

Our retrospective study presents no differences in efficacy or safety profiles of cyclosporine, or tacrolimus in cardiac transplant patients, but it is limited by the small number of patients. Monitored plasma levels were within the target ranges, but drug iatrogeny manifested by progressive evolution to chronic renal disease.

Obtaining an individual predictive pharmacokinetic profile is difficult due to intrinsic variability of drugs and progressive deterioration of renal function (related to age, but also influenced by the nephrotoxicity of the used immunosuppressant agents).

\section{Acknowledgments.}

This paper is partially supported by the Sectorial Operational Programme Human Resources Development financed from the European Social Fund and by the Romanian Government under the contract number POSDRU/89/1.5/S/60782

\section{References}

1. Oellerich M., Amstrong VW. \& Kahan B. et al. (1995) Lake Louise consensus conference on cyclosporin monitoring in organ transplantation: report of consensus panel, Ther Drug Monit. 17, 642-654

2. Kahan BD., Shaw LM. \& Hold D. et al. (1990) Consensus document: Haw's Cay meting on therapeutic drug monitoring of cyclosporine, Clin Chem. 36, 1510-6

3. Levey AS., Coresh J., Greene T., Stevens LA., Zhang YL. \& Hendriksen S., et al. (2006). Chronic Kidney Disease Epidemiology Collaboration. Using standardized serum creatinine values in the Modification of Diet in Renal Disease study equation for estimating glomerular filtration rate. Ann Intern Med. 145(4), 247-254.

4. Miller WG. (2011). Glomerular Filtration Rate. The Importance of Standardized Serum Creatinine in Detecting Kidney Disease. Clinical Laboratory News. 37(12), 10-12.

5. Marqut P., Leger F., Pisano P. \& Billaud EM. (2004). Suivi thérapeutique de la ciclosporine In Suivi thérapeutique pharmacologique pour l'adaptation de posologie des médicaments, (pp 280-293). Paris: Elsevier

6. Tedesco D. \& Haragsim L. (2012). Cyclosporine: A Review, Journal of Transplantation, Article ID 230386, 7 pages. DOI:10.1155/2012/230386

7. Martins L., Ventura A. \& Branco A. et al. (2004). Cyclosporine versus tacrolimus in kidney transplantation: are there differences in nephrotoxicity? Transplant Proc. 36(4), 877879.

8. Ojo AO., Held P.J. \& Port F.K. et al. (2003). Chronic renal failure after transplantation of a nonrenal organ. The New England Journal of Medicine. 349(10), 931-940

9. Ispas M., Chincisan C., Voidăzan S., Mărginean A. \& Dobreanu M. (2012). Chronic kidney disease after treatment with cyclosporine or tacrolimus in heart transplant recipients - experience of a 
Romanian medical center. Revista Română de Medicină de Laborator. 20(2/4), 117-125

10. Castel M.A., Farrero M., Vallejos I., Cardona M., Regueiro A. \& Pérez-Villa F. (2011). Primary immunosuppression and outcome differences after heart transplantation: tacrolimus versus cyclosporine. Transplant Proc. 43(6), 2244-6

11. Meiser B.M., Groetzner J. \& Kaczmarek I. et al (2004). Tacrolimus or cyclosporine: which is the better partner for mycophenolate mofetil in heart transplant recipients? Transplantation. 78(4), 591-8.

12 Baran D.A., Zucker M.J. \& Arroyo L.H. et al. (2011). A prospective, randomized trial of singledrug versus dual-drug immunosuppression in heart transplantation: the tacrolimus in combination, tacrolimus alone compared (TICTAC) trial. Circ Heart Fail. 4(2), 129-37
13. Kobashigawa J.A. (2011). Strategies in immunosuppression after heart transplantation: is less better? Circ Heart Fail. 4(2), 111-3

14. Garlicki M. (2005). Post-transplant diabetes mellitus (PTDM) in heart recipients, Ann Transplant. 10(3), 51-3

15. Simmonds J., Dewar C., Dawkins H., Burch M. \& Fenton M. (2009). Tacrolimus in pediatric heart transplantation: ameliorated side effects in the steroid-free, statin era. Clin Transplant. 23(3), 415-419

16. Morales J.M., Andrés A. \& Dominguez-Gil B. et al. (2005). Ten years of treatment with tacrolimus is related to an excellent renal function, allowing monotherapy in a large proportion of cases: unicentric results of the tacrolimus versus cyclosporine. A European multicentric study in kidney. Transplantation Proceedings. 37(9), 3738-3742 


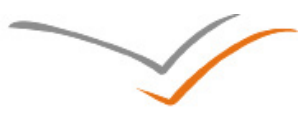

${ }^{1}$ University of Medicine and Pharmacy Targu Mures

${ }^{2}$ County Clinical Emergency Hospital Targu Mures

\begin{abstract}
ABTRACT.
Constrictive pericarditis is an uncommon condition, often of unknown etiology. The diagnosis of constrictive pericarditis can be difficult and is often delayed, because the signs and symptoms of this disease can be falsely attributed to other causes. We report the case of a 62-year-old woman presented with a one year history of progressively worsening dyspnea, peripheral oedema, prominent jugular distension, hepatomegaly, ascites. The patient is known with a history of effusive pericarditis. Blood test showed a normal white cell count, anaemia, raised CRP. Chest X-Ray shows a normal sized heart and without calcification of the pericardium. The echocardiographic exam showed septal bounce-abrupt transient rightward movement, left, right ventricular size decreased-heart tubularin shape, mild atrial enlargement, IVC plethoric and unresponsive to respiration, hepatic veins dilated. Doppler echocardiographic findings were consistent with constrictive pericarditis. Cardiac catheterization showed elevation and equalization of diastolic filling pressures, and dips and plateau configuration of ventricular pressure during diastole (square root sign). Based on these results pericardiectomy was necessary. Constrictive pericarditis was also confirmed at the time of surgery. The pericardium was found with thickening
\end{abstract}

\section{Suciu H}

University of Pharmacy and Medicine Tg. Mureş

38 Gh. Marinescu, Tg. Mureş,

Tel: +40-265-215-551

E-mail: horisuciu@gmail.com of up to $30 \mathrm{~mm}$ in some areas. Histopathological exam showed fibrosis and calcification within the pericardium with no evidence of malignancy or tuberculosis. The patient was discharged 10 days later. At 3 months she had no significant symptoms. Diagnosis of constrictive pericarditis remains challenging. Constrictive pericarditis should be suspected in patients with clinical features of right-sides heart failure. Echocardiography and cardiac catheterisation are important investigation in diagnosis of constrictive pericarditis and avoiding unnecessary treatments.

Keywords: constrictive pericarditis, echocardiography, heart failure

\section{Introduction}

Constrictive pericarditis is an uncommon condition, represents the end stage of an inflammatory process involving the pericardium and causes myocardial compression and restricted filling of the heart. In the past tuberculosis was the most common cause of constrictive pericarditis, and it still remains important in developing countries. In the developed world the cause is most commonly idiopathic, postsurgical, or radiation injury, but also can be caused by viral, bacterial or fungal infection, autoimmune disorders, uremia, neoplasia, posttraumatic, and inflammatory reaction to a foreign body [1] The clinical presentation is usually characterized by clinical signs and symptomps of right-heart failure 
due to pericardial constriction. The diagnosis of constrictive pericarditis can be difficult and is often delayed, because the signs and symptoms of this disease can be falsely attributed to other causes.

\section{Gase presentation}

We report the case of a 62-year-old woman presented with a one year history of progressively worsening dyspnea, peripheral edema, prominent jugular distension, hepatomegaly, ascites. The patient is known with a history of effusive pericarditis. Blood test showed a normal white cell count, anaemia, raised CRP. ECG shows sinus rhythm, normal voltage and nonspecific $\mathrm{T}$ wave abnormalities (negative $\mathrm{T}$ wave in V1-V3). Chest X-Ray shows a normal sized heart and without calcification of the pericardium. The echocardiographic exam showed septal bounceabrupt transient rightward movement, left, right ventricular size decreased-heart tubularin shape (Figure 1), mild atrial enlargement, IVC plethoric and unresponsive to respiration, hepatic veins dilated, mild pulmonary hypertension (PAPs $40 \mathrm{mmHg}$ ) and no pericardial thickening.

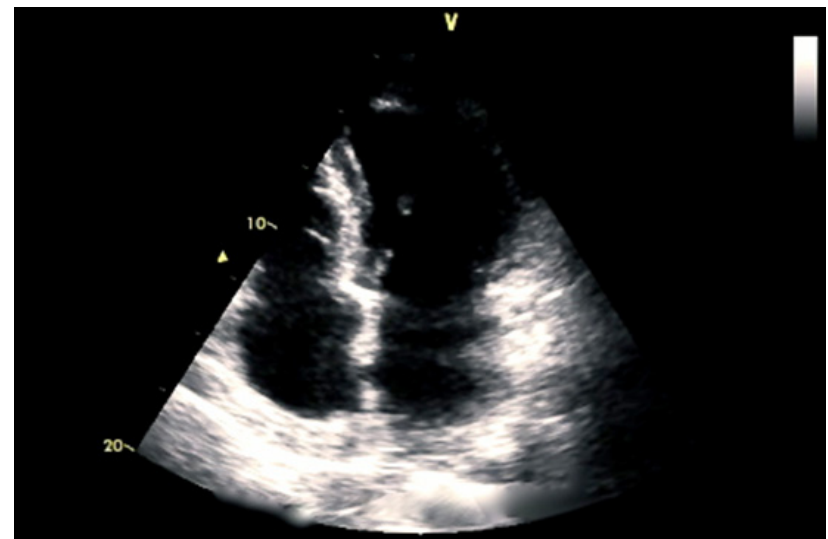

Figure 1 - four chambers echocardiographic view with normal cavities dimensions
Doppler echocardiographic findings showed restrictive mitral inflow $\mathrm{E} / \mathrm{A}>2$, DT $116 \mathrm{msec}$, respiratory changes in the mitral $\mathrm{E}$ velocity $(>50 \%)$ (Figure 2), reversed hepatic venous flow during expiration, high E' velocity $(14 \mathrm{~cm} / \mathrm{sec})$.

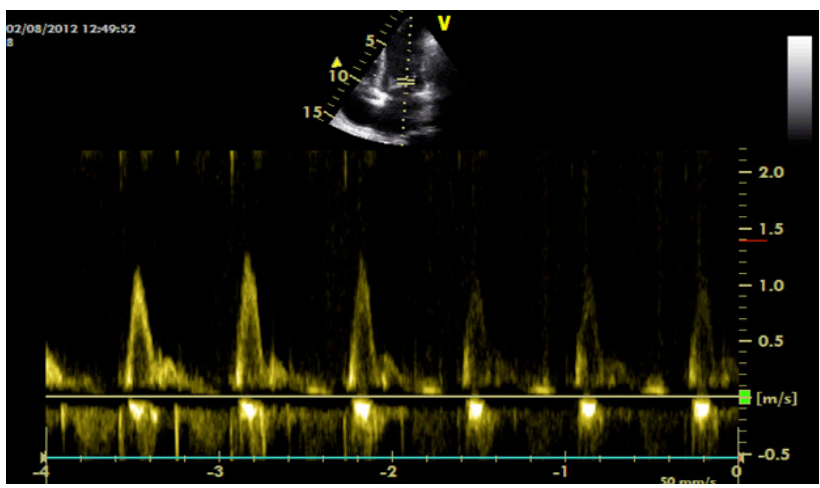

Figure 2 - restrictive mitral inflow $E / A>2, D T 116 \mathrm{msec}$, respiratory changes in the mitral E velocity (>50\%)

A subsequent cardiac catheterization indicated no significant coronary artery and a hemodynamic profile consistent with constrictive, showed elevation and equalization of diastolic filling pressures, and dip and plateau configuration of ventricular pressure during diastole (square root sign) (Figure 3).

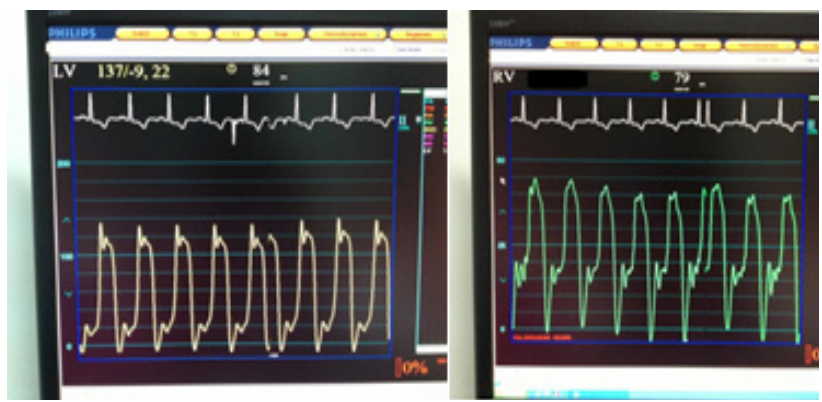

Figure 3 - showed elevation and equalization of diastolic filling pressures, and dip and plateau configuration of ventricular pressure during diastole (square root sign)

Based on these results the final diagnosis was constrictive pericarditis and pericardiectomy was considered necessary. Constrictive pericarditis was also confirmed at the time of surgery. The pericardium was found with thickening of up to 30 
$\mathrm{mm}$ in some areas. Histopathological exam showed fibrosis and calcification within the pericardium with no evidence of malignancy or tuberculosis. Based on histopathological exam, laboratory exams and no significant medical history the diagnosis of idiopathic constrictive pericarditis was made. The patient was discharged 10 days later. At 3 months she had no significant symptoms.

\section{Discussion}

The diagnosis of constrictive pericarditis is still difficult and requires a high degree of clinical suspicion. Constrictive pericarditis is characterized by the thickening of the pericardium by chronic fibrosis resulting in severe diastolic dysfunction with abnormal diastolic filling of the ventricles due to constriction within the pericardial sac. The characteristic hemodynamic pattern consists of rapid ventricular filling in the early stages of diastole that ends when the stiff pericardium limits the filling. At this point all diastolic pressures are elevated and equalized ("diastolic equalization") [2]. Functionally this cause systemic venous congestion and failure of outflow. There are many causes of venous congestion, and due to the difficulty in clinical diagnosis, investigation are vital in making the diagnosis. A prior history of pericarditis , trauma or cardiac surgery makes the diagnosis of CP more likely [3].

Constrictive pericarditis and restrictive cardiomyopathy have some similarities in clinical presentation but their pathophysiology, clinical features, and more importantly therapeutic approach is different, so that the correct diagnosis should be made before taking the therapeutic decision [4]. Echocardiography, cardiac catheterization, CT and MRI are important investigation to establish the correct diagnosis.

There are a number of echocardiographic differences between constrictive pericarditis and restrictive cardiomyopathy, but because they also share important haemodynamic characteristics, they have a number of Doppler characteristics in common. The most important is a restrictive mitral flow or ventricular filling pattern with striking $\mathrm{E}$ dominance and a short deceleration time, due to early rapid filling that is seen in both entities. In establishing the correct differential diagnosis between $\mathrm{CP}$ and RCMP the most important are the chances with respiration of the mitral flow velocity (early diastolic left ventricular filling increases with expiration and reciprocal changes in right-sided Doppler flows) $[3,5]$. The respiratory variation in ventricular filling velocity in RCMP is usually minimal, less than $10 \%$, and in patients with CP may have variations as high as $30-40 \%$. There are both false positive and negative results when examining the respiratory variation of mitral flow velocity to differentiate CP from RCMP. The ventricular filling velocity is highly influenced by preload, when LAP is greatly elevated respiratory variation in this parameter may not be seen in patients with $\mathrm{CP}$, and in case of atrial fibrillation the presence of highly variable RR-interval makes the diagnosis of CP difficult $[3,5]$.

Computed tomographic scanning of the heart in case of CP shows increased pericardial thickness and calcification, but a normal appearance or nonvisualization of the pericardium does not exclude the diagnosis of $\mathrm{CP}$, since pericardial stiffening can occur without calcification.

In some cases invasive cardiac catheterization is required to establish the diagnosis. Elevation and equalization of diastolic filling pressures occur in patients with $\mathrm{CP}$, the dip and plateau configuration of ventricular pressure (square root sign) during diastole corresponds to a rapid early filling aided by augmented suction followed by a hampered further filling caused by rapidly increasing pressures.

In the reported case we did not considered necessary CT exam, because no pericardial thickening and calcification were seen in echocardiographic exam and chest $\mathrm{x}$ ray, and cardiac catheterization was necessary for the diagnosis of constrictive pericarditis. 


\section{Conclusion}

Diagnosis of constrictive pericarditis remains challenging. Constrictive pericarditis should be suspected in patients with clinical features of rightsides heart failure. Echocardiography and cardiac catheterisation are important investigation in diagnosis of constrictive pericarditis and avoiding unnecessary treatments.

\section{Acknowlegments}

This paper is partially supported by the Sectoral Operational Programme Human Resources Development, financed from the European Social Fund and by the Romanian Government under the contract number POSDRU/89/1.5/S/60782"

\section{References}

1. Castañón-González JA., Amézquita-Landeros JA., Velasco-Ortega EC., Deseano-Estudillo JL. \& León-Gutiérrez MA. (2010). Constrictive pericarditis: the story of a constrained heart. $\mathrm{Cir}$ Cir, 78, 336-340

2. Zamorano JL., Bax J., Rademarkers F. \& Knuti J. (2010). The ESC Textbook of Cardiovascular Imaging.

3. Maisch B., Seferovic PM. \& Ristic AD. et al. (2004). Guidelines on the diagnosis and management of pericardial diseases executive summury; the Task force on the diagnosis and management of pericardial diseases of the European society of cardiology. Eur Heart J. 25, 587.

4. Oh JK., Hatle LK. \& Seward JB. et al. (1994).
Diagnostic role of Doppler echocardiography in constrictive pericarditis. $\mathrm{J} \mathrm{Am} \mathrm{Coll} \mathrm{Cardiol}$. 23:154.

5. Tabata T., Kabbani SS. \& Murray RD. et al. (2001). Difference in the respiratory variation between pulmonary venous and mitral inflow Doppler velocities in patients with constrictive pericarditis with and without atrial fibrillation. $J$ Am Coll Cardiol. 37, 1936. 


\section{Autologous conditioned plasma in anterior cruciate ligament reconstruction with hamstrings - short term clinical results}

${ }^{1}$ UMF Tîrgu Mureş, Orthopaedics and Traumatology Department

${ }^{2}$ Clinic of Orthopaedics and Traumatology, Tîrgu Mureş

${ }^{3}$ County Hospital "Dr. Eugen Nicoară“", Reghin

\begin{abstract}
ABTRACT.
Evaluating the early clinical results of anterior cruciate ligament reconstruction using hamstrings autograft, with interference screw on the tibial side (biocomposite interference screw, ConMed-Linvatec) and continuous closed loop fixation on the femoral side (XO-Button, ConMed-Linvatec), with and without intra-articular injection of autologous conditioned plasma (ACP). Our study included 21 patients with chronic anterior cruciate ligament (ACL) ruptures for whom we performed ACL reconstruction with a hamstrings autograft. The mean age was 34 years (range, 25 to 42), 16 patients were men and 5 were women. In 10 cases we performed an intraarticular infiltration of ACP at the end of the surgical intervention. Final evaluation was performed at the end of the 6th postoperative month using the Lysholm scoring system, Tegner activity scale and objective assessment with the RolimeterTM 50A. The Lysholm score was excellent in all cases at 6 months postoperatively, with a mean Lysholm score of 90 for patients without ACP and 91.09 for patients that received ACP; the mean Tegner activity score was also similar pre- and postoperatively for the two groups (from 3.5 and 3.63 for the group without ACP and the group with ACP to 5.6 and 5.72 respectively). Joint laxity measurement was similar for both groups. We found no graft ruptures. We found similar results after ACL reconstruction with and without intra-articular injection of PRP, but further studies are necessary to determine the exact role of these substances in speeding up the recovery process in these cases.
\end{abstract}

Keywords: autologous conditioned plasma, anterior cruciate ligament, reconstruction, hamstrings

Gergely I

University of Pharmacy and Medicine Tg. Mures

$38 \mathrm{Gh}$. Marinescu, Tg. Mureş,

Tel: +40-265-215-551

\section{Introduction}

Given its location and function, the knee is subject to significant forces during physical activity, with approximately 15 to $30 \%$ of sports injuries located at this level. The most common ligament injuries of the knee are complete ruptures of the anterior cruciate ligament (ACL), which usually require surgical treatment followed by a long-term recovery program. If not treated properly, ACL injuries can cause significant functional deficits and may lead to early arthritic changes of the affected joint $[5,9,12]$.

With increased participation in sports activities population-wide, the incidence of anterior cruciate ligament injuries tends to increase. Also the expectations of professional athletes and recreational sports practitioners increased - most of them want to return to their pre-lesional level of activity as soon as possible after surgery.

ACL reconstruction is an efficient treatment of knee joint instability caused by a ruptured anterior cruciate ligament, and had become a relatively common procedure in recent years, with about 6570000 interventions/year (U.S.A.). An ACL deficient knee is associated with an increased rate of meniscal tears and degenerative changes, which is one of the important reasons for ACL reconstruction.

The use of hamstring autografts has recently increased in popularity, among patients as well. With the development and improvement of graft fixation methods, the objective biomechanical and functional results of using semitendinosus-gracilis autografts have matched and even exceeded those of bone- 